\title{
A PROPERTY OF SOME OPEN RIEMANN SUPFACES AND ITS APPLICATION
}

\author{
TADASHI KURODA
}

\section{Introduction}

As L. Sario [8] and the others have shown, an important method to investigate the properties of an open Rientann surface $F$ is the use of an exhaustion of $F$ consisting of compact domains on $F$. But, for the same purpose, the investigation of properties of a non-compact region on $F$ is also important.

Recently Lauri Myrberg [5] gave remarkable results for some harmonic functions and Ullemar [12], [13] gave interesting theorems on a symmetric Fuchsian or fuchsoid group without any elliptic transformation and of genus zero. These results concern with the property of a non-compact region.

Let $G$ be a non-compact domain on $F$ whose relative boundary $C$ consists of at most an enumerable number of compact or non-compact ana'y'ic curves clustering nowhere in $F^{1}$. We can construct an open Riemann surface $\hat{G}$ by the process of symmetrization. There is given an indirectly conformal mapping of $\hat{G}$ on itself which leaves every point on $C$ fixed. The image of a point $\phi \in \hat{G}$ is denoted by $\tilde{p}$, the image of $G$ by $\widetilde{G}$. If $t=x+i y$ is the local parameter at $p \in \hat{G}$, the local parameter at $\tilde{p}$ is given by $t=x-i y$.

In this article, we shall investigate some properties of such a surface $\hat{G}$ and give some remaris on the results obtained by Myrberg and Ullemar as its application.

\section{Properties of $\hat{G}$}

1. We denote by $H B$ or $H D$ the class of single-valued bounded or Dirichict bounded $^{2)}$ harmonic functions respectively. If any function of $H B$ (or $H D$ ) in $G$, which equals to zero on $C$ and is continuous on $G \smile C$, equals identically to zero, we may say that $G$ belongs to the class $S O_{H I}$; (or $S O_{H I}$ ). Further, we call that $G$ belongs to the class $N O_{H i} ;\left(O * N O_{H i}\right)$, if each function of $H B$ (or $H D$ ) in $G$ whose normal derivative at every point on $C$ vanishes and which is continuous on $G \smile C$, reduces to a constant.

First we shall prove the following

Received April 20, 1953.

1) Simply we call such a domain $G$ a non-compact region throughout this article.

2) We may say that the function is Dirichlet bounded in a region if the Dirichlet integral of it over the region is finite. 
THEOREM 1. If $G$ is a non-compact region on an open Riemann surface $F$ with null boundary, then $G \in S O_{H B}, \in S O_{H D}, \in N O_{H B}$ and $\in N O_{H D}$.

Proof. It is an immediate consequence from the maximum and minimum principle that $G \in S O_{H B}$ and $\in S O_{H D}$. We shall give a proof of $G \in N O_{I B B}$.

Let $\left\{F_{13}\right\}(n=0,1, \ldots)$ be an exhaustion of $F$ satisfying the condition $\bar{F}_{0} \subset G$ and $I_{n}$ be the relative boundary of $F_{n}$. We denote by $u$ the harmonic function in $F_{n}-\bar{F}_{0}(n \geqslant 1)$ which equals to zero on $\Gamma_{0}$ and to $\log \mu_{n}$ on $\Gamma_{n}$, where $\log \mu_{n}$ is the harmonic modulus ${ }^{3)}$ of $F_{n}-\bar{F}_{0}$, and by $v$ the conjugate function of $u$. Then, from the definition of the harmonic modulus,

$$
\int_{\Gamma_{n}} d v=2 \pi
$$

Let $\Gamma_{\lambda}$ be the part of a niveau curve $u=\lambda\left(0<\lambda \leqq \log \mu_{n}\right)$ contained in $G$.

If $G \in N O_{H B}$, there exists a non-constant function $U(p)$ of $H B$ on $G$ whose normal derivative $\frac{\partial U}{\partial \nu}$ vanishes at every point on the relative boundary $C$ of $G$. If $D(\lambda)$ is the Dirichlet integral of $U(p)$ taken over the compact open set bounded by $\Gamma_{\lambda}$ and $C$ and consisting of a finite number of compact domains, we have

$$
D(\lambda)=\int_{\Gamma \lambda} U \frac{\partial U}{\partial u} d v
$$

for $\frac{\partial U}{\partial \nu}$ equals to zero on $C$. By the Schwarz inequality, we get

$$
\begin{aligned}
D^{2}(\lambda) & \leqq \int_{\Gamma_{\lambda}} U^{2} d v \int_{\Gamma \lambda}\left(\frac{\partial U}{\partial u}\right)^{2} d v \\
& \leqq M^{2} \int_{\Gamma_{\lambda}} d v \int_{\Gamma_{\lambda}^{\prime}}\left(\frac{\partial U}{\partial u}\right)^{2} d v \\
& \leqq 2 \pi M^{2} \frac{d D(\lambda)}{d \lambda},
\end{aligned}
$$

provided that $|U(P)| \leqq M$, whence it follows that

$$
d \lambda \leqq 2 \pi M^{2} \frac{d D(\lambda)}{D^{2}(\lambda)}
$$

Integrating the both sides from $\lambda=0$ to $\lambda=\log \mu_{n}$, we obtain

$$
\begin{aligned}
\log \mu_{n} & \leqq 2 \pi M^{2}\left[\frac{1}{D_{0}}-\frac{1}{D_{n}}\right] \\
& <2 \pi M^{2} \frac{1}{D_{0}},
\end{aligned}
$$

3) The notion of the harmonic modulus was introduced by Sario [8] and Pfluger [7]. 
where $D_{n}$ is the Dirichlet integral of $U(p)$ taken over $G \cap F_{n}$. Therefore, it is immediate that $\lim _{n \rightarrow \infty} \mu_{n}<\infty$.

On the other hand, it is well known that $\lim _{n \rightarrow \infty} \mu_{n}=\infty$, if and only if $F$ has a null boundary (cf. Kuroda [2]). Thus $G \in N O_{H B}$ if $F$ has a null boundary.

By the similar arguments as above (cf. Kuroda [3], Tsuji [11]), we can prove the fact that $G \in N O_{H D}$ in the case of $F$ with null boundary.

2. Here we shall state a theorem of Myrberg in the following form. ${ }^{4}$

Theorem 2. Suppose that $G \in S O_{H B}$ (or $\in S O_{H D}$ ) and that $U(p)$ is a function of $H B($ or $H D)$ on $\hat{G}$. Then $U(p)=U(\widetilde{p})$ for any $p \in \hat{G}$.

- Proof. Putting $V(p)=U(p)-U(\widetilde{p})$, we can see that $V(p)$ is also a function of $H B$ (or $H D$ ) on $\hat{G}$ and so in $G$ and $V(p)=0$ on $C$. Since $G \in S O_{H B}$ (or $\left.\in S O_{H D}\right), V(p)$ reduces to a constant zero in $\hat{G}$ and hence $U(p)=U(\tilde{p})$.

Now we can prove the following

THEOREM 3. If $G \in S O_{H B}$ and $\in N O_{H R}$, then $\hat{G} \in O_{H B}$, in other words, there exists no non-constant function of $H B$ on $\hat{G}$ and vice versa.

Proof. Let $U(p)$ be a function of $H B$ on $\hat{G}$. By Theorem 2, we get $U(p)$ $=U(\tilde{p})$ on $G$. Hence it is immediate that the normal derivative of $U(p)$ at every point on $C$ vanishes. Since $G \in N O_{H B}$ by the assumption, $U(p)$ must reduce to a constant, which proves the first part of our assertion.

Next we shall prove the converse. Suppose that $G \notin S O_{H B}$. Then there exists a non-constant function $U(p)$ of $H B$ in $G$ which equals to zero on $C$ and is continuous on $G \smile C$. If we define a function $V(p)$ on $\hat{G}$ such that

$$
V(p)=\left\{\begin{array}{rl}
U(p) & \text { for } p \in G \smile C \\
-U(p) & \text { for } p \in \widetilde{G}
\end{array},\right.
$$

$V(p)$ belongs to $H B$ and is non-constant. On the other hand, if $G \notin N O_{I I B}$, there exists a non-constant function $U(p)$ of $H B$ in $G$ whose normal derivatives on $C$ vanish and which is continuous on $G \smile C$. The function

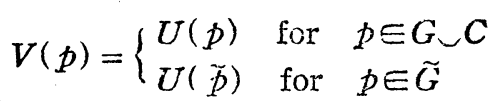

on $\hat{G}$ is a non-constant function of HB. Thus the proof is complete.

By Nevanlinna's theorem [6], for a Riemann surface with finite genus, $F \in O_{I I B}$ (or $\left.\in O_{H D}\right)^{5)}$ is equivalent to that $F$ has a null boundary. Hence we get

4) See Theorem 5.

5) The class $O_{H D}$ means the class of Riemann surfaces on which there exists no non-constant function of $H D$. 
Corollary. If $G \in S O_{I I 3}$ and $\in N O_{I H B}$ and, further, $G$ is finitely connected, $\hat{G}$ has a null boundary.

By the similar arguments as above, we have the followings.

ThEOREM 3 . If $G \in S O_{I I D}$ and $\in N O_{I I D}$, then $\hat{G} \in O_{H n}$, and vice versa.

COROLlaRY. For a finitely connected region $G, \hat{G}$ has a null boundary under the same condition for $G$ as in Theorem 3 .

Remark. Under the condition of Theorem $3, \hat{G}$ has not always a null boundary. This is observed from the example of a Riemann surface, due to Tôki [9], which has a positive boundary and belongs to $O_{I I B}$.

Further, we get

Theorem 4. If $G \in S O_{I I B}$ (or $\left.\in S O_{I D D}\right)$, then $\hat{G} \in O_{A B}\left(\right.$ or $\left.\in O_{A D}\right)$. $^{6}$

Prcof. Let $f(p)=U(p)+i V(p)$ be a single-valued bounded (or Dirichlet bounded) analytic function on $\hat{G}$. Then, obviously, $U(p) \in H B$ (or $\in H D$ ) and $V(p) \in H B$ (or $\in H D$ ). By Theorem 2, $U(p)=U(\widetilde{p})$ and $V(p)=V(\tilde{p})$ for any $p \in \hat{G}$. Therefore, the differential $d f$ must equal to zero at every point on $C$. Thus $f(p)$ reduces to a constant and so $\hat{G} \in O_{A B}$ (or $\in O_{A D}$ ).

Remark. Mori [4] pointed out the fact that, for a simply connected region $G$, the converse of Theorem 4 holds good.

\section{Applications}

3. Now we shall prove Myrberg's theorem [5] applying theorems stated above. We sippose that a non-compact region $G$ is simply connected. Then $G$ can be mapped on the upper half $z$-plane one to one conformally such that a point on the relative boundary $C$ of $G$ corresponds to $z=\infty$. The ideal boundiry of $G$ corresponds to a bounded closed linear set $E$ on the real axis of the $z$-plane. If we denote by $\Omega$ the complementary domain of $E$ with respect to the whole $z$-plane, $\Omega$ is equivalent to $\hat{G}$ conformally.

By Mori's remark [4], $E$ is of measure zero, if and only if $G \in S O_{I I B}$. And, if we notice Ahltors-Beurling's theorem [1], it is easily seen that $\Omega$ is of span zero if ard only if $G \in S O_{H D}$. Hence we have the following by Theorem 2 .

Theonem 5 (Myrberg [5]). Let E be a bounded closed set on the real axis in the z-plane and let $U(z)$ be a function of $H B$ in the complementary domain $\Omega$ of $E$ with respect to the z-plane. If $E$ is of measure zero, then $U(z)=U(\bar{z})$.

6) We denote by $O_{A B}$ (or $O_{A D}$ ) the class of Riemann surfaces on which there exists no nonconstant singie-valued tounded (or Dirichlet bounded) analytic function. 
Ant if $V(z)$ is a funtion of HD in $\Omega$ and the spain of $\Omega$ equals to zero, ihen $V(z)=V(z)$.

4. We shall use the notation as in the previous section and prove the following

Theorem 6. A simiply connected region $G$ belongs to $N O_{u l}$, if and only if the set $E$ is of logarithmis capacity zero.

Proof. If $E$ is of logarithmic capacity zero, then, of course, $\Omega \in O_{I I H}$ and hence $\hat{G} \in O_{i I j}$. Thus the sufficiency of our condition is obvious from Theorem 3. We shall state the proof of the necessity.

For its purpose, it is sufficient to prove that, if the logarithmic capacity of $E$ is positive, there exists a non-constant function of $H B$ in the upper half $z$ plane whose normal derivative at every point on the real axis vanishes excluding the set $E$. Since the logarithmic capacity of $E$ is positive, there exist two closed subsets $E_{1}$ and $E_{2}$ of $E$ such that they are disjoint each other and their logarithmic capacities are both positive. We denote by $\Omega^{\prime}$ the complementary domain of $E_{1} \cup E_{2}$ and choose an exhaustion $\left\{Q_{n}\right\}(n=1,2, \ldots)$ of $\Omega^{\prime}$ as follows:

The boundary $I_{n}$ of $\Omega_{n}$ consists of two classes $\Gamma_{n}^{(1)}$ and $\Gamma_{n}^{(2)}$ of analytic closed curves such that $\left\{\Gamma_{n}^{(1)}\right\}(n=1,2, \ldots)$ clusters to $E_{1}$ and $\left\{\Gamma_{n}^{(?)}\right\}(n=1,2, \ldots)$ to $E_{2}$, and $\Omega_{n}$ is symmetric for the real axis.

Let $\omega_{n}$ be a harmonic function in $\Omega_{n}$ which equals to zero on $\Gamma_{n}^{(1)}$ and to 1 on $\Gamma_{n}^{(2)}$. Then we can easily find a suitable subsequence, say again $\left\{\omega_{n}\right\}$, such that it converges to a non-constant limiting function $\omega$ which is harmonic in $\Omega^{\prime}$.

Since each function $\omega_{n}$ is symmetric for the real axis, the limiting function $\omega$ is also symmetric for the real axis. From this fact, the normal derivative of (1) vanishes at every point on the real axis except all points of $E_{1}$ and $E_{2}$. Thus our proof is complete.

In the above proof, choosing a suitable subsequence of $\left\{\omega_{n}\right\}$, we can get a limiting function $\omega$ Dirichlet bounded in $\Omega^{\prime}$. Hence we have easily

THEOREM 6 . The simply connected non-compact region belongs to $N O_{I I D}$, if and only if $E$ is of logarithmic capacity zero.

As we can map the whole $z$-plane on the whole $w$-plane by a linear transformation one to one conformally such that the upper half $z$-plane corresponds to the unit circle $|w|<1$ and the ideal Doundary of $G$ corresponds to a closed set $E$ on the circumference $|w|=1$. Hence we can get the following.

THEOREM 7. Let $E$ be a closed set on the circumference of the unit circle on the complex plane and let $C$ be the complementary set of $E$ with respect to the circumiference. In order that any function in the unit circle belonging to 
$H B$ or $H D$, whose normal derivative at every point on $C$ vanishes, reduces to a constant, it is necessary and sufficient that the set $E$ is of logarithmic cajacity zero.

In fact, it is sufficient to consider the case where $E$ is not identical to the circumference of the unit circle. Considering the complementary domain of $E$ with respect to the whole plane as an open Riemann surface and using Theorems 6 and $6^{\prime}$, we arrive at the required.

Renark. Myrberg [5] proved only the sufficiency of the condition for $H D$. Further, the following complete form of Myrberg's theorem is also easily obtained.

THEOREM 7'. Under the same notation as in Theorem 7, any single-valued positive harmonic function in the unit circle whose normal derivative at each point on $C$ vanishes, reduces to a consiant, if and only if $E$ is of logarithmic capacity zero.

For, there exists a non-constant function of $H B$ in the unit circle whose normal derivative at every point on $C$ vanishes, if the logarithmic capacity of $E$ is positive. Hence there exists a single-valued non-constant positive harmonic function in the unit circle whose normal derivative at every point on $C$ vanishes. Thus the necessity of cur condition is obtained. The sufficiency is nothing but the result obtained by Myrberg.

5. From Theorems 6 and $6^{\prime}$, it is immediately seen that, for a simply connected region $G, G \in N O_{I B B}$ is equivalent to $G \in N O_{H D}$. Further, we have

TheOREM 8 (Tsuji [10]). Let $G$ be a simply connected domain bounded by an analytic Jordan curve $C$ and let $E$ be a closed set on $C$ with logariihmic capacity zero. If we map $G$ on the unit circle one to one conformally and denote by $E^{\prime}$ the closed set on the circumference of the unit circle corresponding to $E$, then $E^{\prime}$ is of logarithmic capacity zero.

In fact, the complementary domain of $E$ with respect to the whole plane may be considered as an open Riemann surface with null boundary. Hence, by Theorem 1, we can see that $G \in N O_{H B}$ considering the set $E$ as the ideal boundary of this Riemann surface. Thus our assertion is an immediate result of Theorem 6.

Remembering Mori's remark stated in $\S 3$ and using Theorem 6 , we have

THEOREM 9. The class $N O_{I I B}$ of simply connected regions is a proper subclass of $S O_{I I B}$ of simply connected regions.

6. Consider a finite or an enumerable number of circular open $\operatorname{arcs}\left\{\alpha_{i}\right\}$ $(i=0,1,2, \ldots)$ in the unit circle $|z|<1$ which are orthogonal to the circumfer- 
ence $|z|=1$ and disjoint each others in $|z|<1$ and denote by $D_{0}$ the simply connected domain in $|z|<1$ bounded by $\left\{\alpha_{i}\right\}(i=0,1,2, \ldots)$ and the closed set $E$ on $|z|=1$. If $\widetilde{D}_{0}$ is the reflection of $D_{0}$ with respect to an arc of $\left\{\alpha_{i}\right\}$, say $\alpha_{0}$, then the domain $D_{0} \cup \alpha_{0} \cup \widetilde{D}_{0}$ is a fundamental domain of a symmetric Fuchsian or fuchsoid group $\$$ without any elliptic transformation and of genus zero. Conversely, such a symmetric group has a fundamental domain as stated above.

We denote by $\left\{\tilde{\alpha}_{i}\right\}(i=0,1,2, \ldots)$ the boundary arcs of $\widetilde{D}_{0}$ being equivalent to $\left\{\alpha_{i}\right\}(i=0,1,2, \ldots)$ for $\$$. Of course, $\tilde{\alpha}_{0}$ is identical to $\alpha_{0}$. Identifying the equivalent points on $\alpha_{i}$ and $\tilde{\alpha}_{i}(i=1,2, \ldots)$, we get an open Riemann surface $\hat{D}$. This surface $\hat{D}$ can be decomposed by a relative boundary $C$ into two parts $D$ and $\widetilde{D}$, each one of which is the image of the other by an indirectly conformal mapping. And $D \smile C$ (or $\widetilde{D} \smile C$ ) is conformally equivalent to $D_{0} \smile \bigcup_{i=0}^{\infty} \alpha_{i}$ (or $\widetilde{D}_{0} \cup \bigcup_{i=0}^{\infty} \widetilde{\alpha}_{i}$ ).

We shall show the following

THEOREM 10. If the set $E$ is of logarithaic cafacity zero, then $\hat{D}$ has a mull boundary.

Proof. The complementary domain of $E$ with respect to the whole $z$-plane can be considered as an open Riemann surface with null boundary. Hence, by Theorem 1, the domain $D_{0}$ and so $D$ belongs to $S O_{H I}$ and to $N O_{H I}$. By the Corollary of Theorem $3, \hat{D}$ has a null boundary.

Remark. The necessary and sufficient condition in order that $\hat{D} \in O_{A i}$ or $\in O_{A n}$ was obtained by Ullemar (=Uskila) [12], [1:]. His results can be proved easily by using Theorem 4 and by remembering the remark stated in $\S 3$.

\section{References}

[1] L. Ahlfors-A. Beurling. Conformal invariants and function-theoretic mull-sets, Acta Math. 83 (1950), pp. 101-129.

[2] T. Kuroda, On the type of an open Riemann surface, Proc. Jap. Acad., 27 (1951), pp. $57-60$.

[3] $T$. Kuroda, Some remarks on an open Riemann surface with null boundary, Tohoku Math. Journ., (2) 3 (1951), pp. 182-186.

[4] A. Mori, A remark on the class $O_{I^{\prime}}$ of Riemann surfaces, Kòdai Math. Sem. Rep., (1952), pp. $57-58$.

[5] L. Myrberg, Bemerkungen zur Theorie der harmonischen Funktionen, Ann. Acad. Sci. Fenn., A.I. 107 (1952), 8 pp.

[6] R. Nevanlinna, Sur l'existence de certaines classes de différentielles analytiques, C. R. 228 (1949), pp. 2002-2004.

[7] A. Pfluger, Über das Anwachsen eindeutige analytischer Funktionen auf offenen Riemannschen Flächen, Ann. Acad. Sci. Fenn., A.I. 64 (1949), 18 pp. 
[ 8 ] L. Sario, Über Riemannschen Fläche mit hebbarem Rand, Ann. Acad. Sci. Fenn., A.I. 50 (1948), 79 pp.

[ 9 ] Y. Tôki, On the classification of open Riemann surfaces, Ôsaka Math. Journ., 4 (1952), pp. 191-202.

[10] M. Tsuji, Beurling's theorem on exceptional sets, Tôhoku Math. Journ., (2) 2 (1950), pp. 113-125.

[11] M. Tsuji, Some theorems on open Riemann surfaces, Nagoya Math. Journ., 3 (1951), pp. 141-145.

[12] L. Uskila, Über die Existenz der beschränkten automorphen Funktionen, Arkiv för Mat., 1 (1949), pp. 1-11.

[13] L. Ullemar, Über die Existenz der automorphen Funktionen mit beschränkten Dirichletintegral, Arkiv för Mat., 2 (1952), pp. 87-97.

Mathematical Institute, Nagoya University 